\title{
Just How Final Are Today's Quantum Structures?*
}

\author{
P. Busch \\ Department of Mathematics, The University of Hull
}

\begin{abstract}
I present a selection of conceptual and mathematical problems in the foundations of modern physics as they derive from the title question.
\end{abstract}

'Quantum theory' is widely believed to be the ultimate framework for the description and explanation of physical experience. Daring as such a 'finality prognosis' may be, any attempt at its justification will, I believe, invariably lead to qualifications: there seems to be no consensus as to how general the formal framework has to be, nor is there any commonly accepted physical interpretation, including the question whether it is necessary or even possible to go beyond the minimal, probabilistic interpretation. Moreover, there are and have always been - some 'clouds on the horizon', that is, some challenging questions concerning the range of applicability of quantum theory. There is thus good reason to rephrase the finality belief into a (hopefully) tractable programme: to determine those elements of the theory that can defendably be regarded as necessary parts of any future theoretical physical framework. This is the foundational route of exploring the scope of the present quantum theory, and I will list some of the ensuing specific problems. The other line of determining the limits of the theory lays, of course, in its application in new areas of experimental research.

1. Hilbert space framework. The significance of the Hilbert space structure of quantum theory has been largely clarified through the quantum logic approach, features of the proposition lattice being exhibited as necessary, apriori conditions of any empirical scientific language. However, the existence of incommensurable sets of propositions or of physical systems with or without superselection rules must be regarded as contingent facts in this approach. Also, the underlying assumption of ideal measurements of sharp observables can be challenged as an unfounded idealisation, so that a revision of the programme could be envisaged that is based on the more general structure of effect algebras. Finally, a fundamental physical understanding of the selection of the complex field seems to be lacking.

2. Quantum-Classical Connection. It is possible to formulate conditions (such as restrictions of the set of states, or superselection rules) under which

*Contribution to "Springer Forum: Quantum Structures - Physical, Mathematical and Epistemological Problems", Liptovsky Jan, 1 September 1998. 
a quantum system will appear to display (approximately) classical behaviour. But a theoretical explanation of why, and under what circumstances, such classicality conditions come to be satisfied seems to be lacking. In other words, the emergence of (approximately?) classical properties in systems of increasing size or complexity has remained unexplained, and this fact has led some researchers to question the universal validity of quantum theory. In my opinion, an approach based on decoherence and environment-induced superselection seems to depend on the feasibility of a universal state vector/many minds interpretation of quantum theory. On such an interpretation, decoherence would guarantee the consistency of the descriptions of physical events given by different observers; but due to the global entanglement this would come at the expense of a relativised notion of objectivity where 'facts' would appear to have a rather transient nature. On a perhaps more (or more naive?) realistic view, the objective description of such classical behaviour as deterministic phase space evolution requires that on an appropriate macroscopic scale, conjugate dynamical variables have to be coexistent and thus jointly measurable, so that their quantum mechanical representatives must be unsharp observables. Thus I think that the fuzzy probability theory recently developed by Bugajski and Gudder may offer a natural unifying framework for a systematic study of the quantum-classical connection.

3. Quantum measurement problem. This is the question of whether and how the stochastic evolution of states in a measurement, viewed as a physical process subject to the laws of quantum theory can be reconciled with the determinism of the unitary Schrödinger dynamics, which does not allow the entanglement of states corresponding to different outcomes to be broken up. The fact that pointer observables, as macroscopic, classical quantities do conform with an objective description implies that their quantum representations are unsharp observables [cf. 2. above]. Thus a relaxation of the concept of objectification is needed that is applicable to unsharp pointers, and the question remains whether 'unsharp objectification' opens a way to solving the measurement problem.

4. Quantum theory and relativity. Special or general relativistic spacetime is a particular instance of a classical domain which turned out notoriously hard to unify with quantum structures. The formal problems of relativistic quantum field theory as well as the fundamental conceptual difficulties encountered in quantum gravity and quantum cosmology remain the most challenging fields of investigation for foundational research into quantum structures.

5. Axiomatics of time evolution. The measurement problem and the problem of time in quantum cosmology require a review of the assumptions underlying the unitary evolution law for closed quantum systems. A closed system is a highly idealised construct, as it becomes more and more difficult with increasing size of systems to shield them from their environment. Thus, it appears that the only conceivable 'large' closed system is the whole universe. Are the conditions of the theorems of Wigner and Kadison on unitary representations of dynamical maps applicable in this singular case?

6. Operational quantum theory and quantum information. On a more 'down to earth' note, quantum mechanics and measurement theory are presently find- 
ing exciting applications in the newly emerging field of quantum information processing. The rich structure of quantum state spaces, which encompass the phenomena of superposition and entanglement, seems to open up entirely new perspectives for enhancing the capacities of communication channels as well as the security of codes. This calls for a systematic development of quantum information theory which takes into account the full structure of quantum probability theory. I see a particularly striking observation in the fact that for certain purposes, measurements of unsharp quantum observables are more efficient than sharp measurements.

7. Quantum theory for individual objects. Is quantum theory 'nothing more' than a statistical theory, or could the referent of a quantum state be a single object? This question reflects a division of the quantum physics community into two distinct 'cultures', corresponding to the two options it addresses. The task of justifying an interpretation that goes beyond a minimal probabilistic interpretation amounts to the task of solving all the quantum puzzles and paradoxes that have made the foundations of quantum mechanics such an exciting enterprise throughout the history of this theory. The needs of quantum cosmology, and also the most recent developments in the experimentation with single microsystems, certainly encourage the search for a sound individual interpretation. Perhaps the answer lies in a fuzzy quantum logic, complemented with an unsharp quantum ontology adapted to a picture of a fuzzy, or soft micro reality. 